\title{
A preliminary evaluation of some language aspects in normal bilingual Egyptian kindergarten children
}

\author{
Azza A. Aziz, Dalia M. Osman, Aisha F. Abdel Hady and Samar Maged ${ }^{*}$ (1)
}

\begin{abstract}
Background: Bilingualism is the phenomenon of speaking and understanding two languages. The increased representation of bilingual populations calls for in-depth studies of bilingualism. The aim of this work is to devise an assessment protocol that could help in evaluating some aspects of Arabic and English languages acquired in typically developing bilingual preschool Egyptian children as a preliminary attempt to comprehend the language profiles of both languages in the studied group. The study was conducted on 80 bilingual Egyptian preschool male and female children exposed to both Arabic and English languages. Their age ranged from 4 to 4 years and 11 months from international English schools with regular attendance and of normal Arabic language development. All were subjected to the designed Bilingual English-Arabic semantic test, and narrative assessment and their parents and teachers filled in the questionnaires addressing the variables affecting both languages' acquisition.
\end{abstract}

Results: Bilingual English-Arabic Semantic and Narration Tests revealed that children under the study had variable development in some aspects in semantic and narration between both languages and according to their age.

Conclusions: Data gathered from the study can be considered a preliminary nucleus for identification of the bilingual children's normative data to be able to pick up the cases with language impairment of bilingual children and help in making diagnostic decisions and planning for future intervention.

Keywords: Bilingualism, Bilingual English-Arabic Test, Semantic development, Narration

\section{Background}

Hamers and Blanc [1] defined bilingualism as "the state of a linguistic community in which two languages are in contact with the result that two codes can be used in the same interaction." Bilingualism has recently become a common condition. This was seen in a study done in the USA which extracted the appropriate numbers and percentage of bilingualism by tabulating every year those who spoke a language other than English, as well as English, and showed a steady increase of the percentage of bilinguals between 1980 and 2016, practically a doubling of the number [2].

\footnotetext{
*Correspondence: Samarmaged2@yahoo.com

Phoniatric Department, Faculty of Medicine, Cairo University, Giza 11865 Egypt
}

Bilingualism is classified into simultaneous bilingualism which applies to children who develop two languages equally or nearly equally through exposure to both and frequent opportunities to use both and successive bilingualism which applies to children who are learning their second language after their first language has been established [3].

Bilingualism is a valuable skill that allows children to use more than one language to experience the world and learn about it. There are many advantages of bilingualism including communication advantages as it provides wider communication networks, literacy in two languages, and metalinguistic awareness of the content and meaning of language; cultural advantages as it provides broader enculturation, the process by which an individual learns the traditional content of a culture and assimilates its 
practices and values and deeper multiculturalism, a social perspective that is committed to publicly recognizing and respecting many cultures and cultural identities; cognitive advantages as it improves thinking, memory, and brain plasticity; character advantages as it raises self-esteem and security in identity. The effect on cognitive performance is to enhance executive functioning and to protect against the decline of executive control in aging, in addition to financial advantages as it increases employment opportunities which results in economic benefits. On the other hand, the effect on linguistic performance is generally seen as a deficit in which bilingual children control a smaller vocabulary than their monolingual peers, there are two languages that are constantly active, and it might lead the verbal skills of a bilingual child to be generally weaker than those noted in monolingual peers speakers of the language [2].

Bilingual children have certain characteristics during their development. Almost all bilingual children go through a stage in which they mix their two languages frequently by inserting elements from the dominant into the weaker language. This phenomenon is called code switch, a specific character of bilingual children, and this mixing is probably due to limited bilingual proficiency [4].

The most common second language acquired in Egypt is the English language. Up to our knowledge, there are few studies examining the language characteristics in both Arabic (the mother tongue) and English (the second language) of normal bilingual Egyptian children. This raised the priority interest in studying this group/ category of population to highlight their normal language features across various language domains. This might assist phoniatricians in assessing bilingual children and in picking up the cases who have or those who are at risk of language impairment in their two languages.

\section{Aim of the work}

The aim of this work is to devise an assessment tool that could help in evaluating some aspects of Arabic and English languages acquired in typically developing bilingual preschool Egyptian children as a preliminary attempt to comprehend the language profiles of both languages in the studied group.

\section{Methods}

\section{Subjects}

This study was conducted on eighty bilingual Egyptian preschool male and female children exposed to both Arabic and English languages. Their age ranged from 4 years to 4 years and 11 months. Participants of this study were divided into two groups according to their age: group A included 47 children with an age range from 4 years to less than 4 years and 06 months old and group B included 33 children with an age range from 4 years and 06 months to 4 years and 11 months old. Group A and group $\mathrm{B}$ were selected from the children in two English private international schools. The two groups were gender and socio-economic standard matched. A number of 5 children were excluded from the study as they had delayed language development which was appeared clearly while performing the test and then reported by their teachers. This study was conducted in the period from December 2018 to June 2019. The protocol of assessment was approved by Cairo University and the department ethical committee.

\section{Inclusion criteria for the study group}

1. Children under the study were recruited from international English schools with regular attendance.

2. The language of instruction at school is English (second language). The language of instruction at home is both Arabic (primary language) and English (secondary language).

3. The time of exposure to secondary language was an average of $5 \mathrm{~h}$ daily at school or at nursery with very limited exposure to primary language, only in Arabic classes, while at home, more exposure time to primary native language than secondary language.

4. The children included in the current study were enrolled in KG1 and KG2 and aged between 4 years and 4 years and 11 months old.

5. Children of Arabic-speaking language parents who are of normal Arabic language development reported by their parents and teachers to have normal language development and without any apparent features that may cause delayed language development (normal hearing, no signs of cognitive delay, neurological or psychiatric disorders).

\section{Exclusion criteria for the study group}

1. Children below 4 years old or older than 5 years old

2. Monolingual children with only exposure to the primary language

3. Children with non-Arabic-speaking parents

4. Children with a history of delayed language development

5. Children with phonological disorder

6. Children with a history of hearing impairment

7. Children with a history of cognitive delay

8. Children with a history of psychiatric disorder

9. Children with a history of neurological disorders 


\section{Methods}

The Bilingual English-Arabic Test (BEAT) used in the current study was specifically designed in this study to be conducted in two languages: Arabic and English for each child. The test was guided by Bilingual English Spanish Oral Screener-Experimental Version (BESOS) by Peña [5], Bilingual English Spanish Assessment (BESA) by Peña [6], and Dynamic Assessment by Gutierrez-Clellen and Pena [7]. The researchers designed the assessment protocol after reviewing the literature and the available Egyptian language assessment tools addressing the age range under the study such as references of APPLE TOOL by Osman [8], REAL Scale by Osman [9], Pls 4 by Abu Haseeba et al. [10], The Arabic language test revised by Rifaie et al. [11], and Assessment of Narrative Skills in Preschool Children by Kamal et al. [12]. The researchers selected the items to be appropriate for the age under the study and to be suitable for the Egyptian culture. The pictures used were drawn and specifically designed during the study by a professional artist. Then, a pilot study was carried out on a number of 10 children to ensure that the whole items of the test are comprehended by the children and to evaluate the applicability of the test. The pilot study revealed that some items needed to be modified such as 1 item in the characteristic property task as the instructions were not so clear and 2 items in the categorization task, 1 item in the linguistic concept task was removed as it was higher than children's abilities and needed to be more comprehended by the children. In addition, some of the drawn pictures in a single item in the feminine and masculine task, a single item in the analogy and one picture in the first story (the doll) were changes as they were not so clear and contained too many details causing distraction to some of the children in the pilot study. The test was applied first in the Arabic language for both semantic and narration sections as it is the mother tongue of the children followed by testing of the second language (English) for both semantic and narration sections (see Additional file 1). The average time taken for both semantic and narration in each language was $20 \mathrm{~min}$.

\section{The semantic assessment section}

The semantic section is divided into seven subtests that target categorization, characteristic properties, functions, linguistic concepts, similarities and differences, feminine and masculine, and analogies. The previous 7 selected items were based on what was found in the literature describing the acquisition of semantics in both languages Arabic (L1) and English (L2) in order to tap into how Egyptian bilingual children organize and access their lexical system. The selected tasks were guided by studies such as Bilingual English Spanish Oral Screener-Experimental Version (BESOS) by Peña [5] and Bilingual English Spanish Assessment (BESA) by Peña [6]. The average time taken to apply the semantic subtests was $10 \mathrm{~min}$ in each language (Table 1).

Scoring of the semantic test was designed as follows:

In the receptive sections of subtests, the child earned a score of 2 when the child responded correctly to the given items in the same language used without the use of any verbal prompt with the other language and earned a score of 0 when the child responded incorrectly to the given items in the same language used, while in expressive sections in the subtests, the child was given a score on a scale from 0 to 2 . He earned a score of 2 when he responded correctly to the given items in the same language used without the use of any verbal prompt with

Table 1 Items of the semantic section of the Bilingual English-Arabic Test by Maged et al. [13]

\begin{tabular}{|c|c|c|}
\hline Subtest & Number of items in each subtest & $\begin{array}{l}\text { Total scoring } \\
\text { of each } \\
\text { subtest }\end{array}$ \\
\hline $\begin{array}{l}\text { 1. Categorization (by identifying an item from a certain category; then labeling the category } \\
\text { of certain items) }\end{array}$ & 8 receptive items and 8 expressive items & 32 \\
\hline $\begin{array}{l}\text { 2. Characteristic property (by identifying the correct item according to a description given, } \\
\text { then describing the object and its attributes) }\end{array}$ & 5 receptive and 6 expressive items & 22 \\
\hline 3. Function (by identifying and labeling an object function provided by picture) & 5 receptive and 5 expressive & 20 \\
\hline $\begin{array}{l}\text { 4. Linguistic concept (by identifying some linguistic concepts like features, time, color, } \\
\text { number, function, and shape) }\end{array}$ & 9 receptive items only & 18 \\
\hline $\begin{array}{l}\text { 5. Similarities and differences (by giving one similarity and one difference between two } \\
\text { objects provided by pictures) }\end{array}$ & 5 expressive items only & 10 \\
\hline $\begin{array}{l}\text { 6. Feminine and masculine (by identifying and using subjective, objective pronouns and } \\
\text { possession) }\end{array}$ & 3 receptive and 3 expressive items & 12 \\
\hline 7. Analogy (by labeling the relationship between object pairs provided by pictures) & 6 expressive items only & 12 \\
\hline
\end{tabular}

Note. The table "items of semantic section of bilingual English-Arabic Test" shows the subtests that were tested in the semantic section of the Bilingual English-Arabic Test (BEAT), the number of items in each subtest, and the total scoring of each subtest 
the other language. The child earned a score of 1 when he responded correctly in the other language to the given items and the child earned a score of 0 when he responded incorrectly to the given items in the same language used. Then, the total semantic test was computed by adding the scores obtained by each child in all the semantic subtest scores. The total score of the whole semantic assessment test was 126 for each language separately.

\section{The narrative assessment section}

The section was guided by a study such as Dynamic Assessment by Gutierrez-Clellen and Pena [7]. The narrative assessment section was assessed by analyzing the responses of each bilingual child by using two stories.

First story (the doll) The first story titled the doll was used to study the children's narrative skills on the microand macrostructure levels. The elicitation method of testing was through the story retelling method. It contains 23 colored pictures one in each page. The story was wordless. It consists of 23 events. The children were asked first to look at the pictures and listen carefully to the story told by the assessor. Then, the children were asked to retell the story in both languages one at a time. The children's responses were audiotaped and then analyzed by the assessor using the following protocol on the macrostructure and microstructure levels.

On the macrostructure level The macrostructure included story frame features and narrative productivity.

Story frame features Six major items of narrative elements were used to evaluate the child's ability to put proper outline for story features starting by giving an introduction, the orientation of character, 10 actions, 1 setting, 1 time, and finally giving a conventional story ending (coda). Scoring here the child was given a score on a scale from 0 to 2 . Two points for mentioning an item in the same language, one point for mentioning an item in the other language, and zero for its absence; then, the points were calculated in order to give the total story frame score which was found to be 12 .

Narrative productivity Narrative productivity consists of three main components: the total number of words, the mean length of utterances, and the ratio of the number of different words spoken over the total number of words (type token ratio) [12]. The total number of words is defined as the total number of words uttered during retelling by each child, while the mean length of utterance (MLU) is a measure of the utterance length. The number of words in the five longest utterances was counted each separately. The average number of words in these utterances was calculated to give us the value of the MLU. When MLU is high, this means that the child can handle a more complex sentence and type token ratio (TTR) which is calculated by counting the number of different words only (any repeated word was not counted) in ratio to the total number of words uttered by the child. The TTR is considered a sensitive indicator for language development in which reflects the variation in the vocabulary used and expresses the complexity of the children's narrative language [14].

On the microstructure level The microstructure included language structure (specificity that means words that give more details such as intensifiers, words referring to physical and emotional state), temporality (which means the subjective progression of timeeg the word "after"), connectives (which means using certain words connecting sentences to give appropriate meaning such as "and," "and so," and "therefore") and reference clarity (which means retelling the content to be easy to be understood with appropriate grammatical properties such as telling presupposed reference using definite article and noun), phrase structure (adjective, adverb, negation, and interrogative phrase), noun form (regular and irregular plurals, possession, colors, and conjoined noun), and verb form (present tense, past tense, future tense, auxiliary verb (in English)). Scoring for phrase structure, noun, and verb was coded for the presence of an item in either language or its absence. Two points were given to the child for mentioning an item in the same language in which the story was told, one point for mentioning an item in the other language, and zero for the absence of the item. Then, the total score of the child was calculated in this story in each language separately.

The second story (The boy and his grandpa) The second story titled The boy and his grandpa was used to assess strategies used by bilingual children such as code mixing (code mixing is when bilingual children mix words from two languages in the same sentence, for example in the story (ice cream chocolate زواع انا)), while code switching is when the language is arranged structurally and grammatically in the other language, for example, the child says I want a chocolate ice cream when asked in Arabic or when asked to repeat the sentence that was given in the Arabic language. This second story contains 10 colored pictures. The story was wordless. It consists of 23 events. This strategy was elicited through asking the children to repeat four sentences that were said by the boy in a loud voice to help his grandpa with hearing difficulty to hear them clearly. Then, analyze the number of errors made in the repeated sentence and count the number of switching 
made by the children. The number of words in each of the four sentences ranged from 6 to 10 words. Responses of the children were audiotaped and then scored as follows.

Sentence repetition The child was given a score on a scale from 0 to 4 . The child earned a score of 4 when he responded correctly by repeating the whole sentences with the same words used and in the same order without any change in sentence structure and in the same language used. The child earned a score of 3 when he produced one error (omitted a word, change the order of words, substitute a word by another one in the same or other language). The child earned a score of 2 when he produced two errors. The child earned a score of 1 when he produced three errors. The child earned a score of 0 when he produced 4 errors. Then, the total score of the child was calculated in this story in each language separately.

Code switch It was calculated by counting the number of code switching which is the number of correct responses that were supplied by the child in the other language while the children retell the story. The code switch item reflected the dominant language and proficiency of a certain language over the other for each child.

\section{Results}

This study was conducted on eighty Egyptian bilingual children of equal gender 40 males and 40 females (group A contains 23 males and 24 females, group B contains 17 males and 16 females). Their ages ranged from 4 to $4: 11$ years; forty-seven children aged from 4:0 to $<4: 50$ with a percentage of $58.8 \%$ and thirty-three children aged from 4:5 to $4: 11$ years with a percentage of $41.2 \%$. The mean age was 4:43 years \pm 0.265 SD. Statistical analysis of the results includes comparative and reliability studies which were done for statistical analysis of the results as shown below.

\section{Bilingual English-Arabic semantic assessment test (Tables 2, 3, 4, and 5)}

Group B showed only higher performance with a significant difference in Arabic similarity and difference task.

The two groups showed no significant difference regarding all test item scores including categorization, characteristic property, function, linguistic concepts, analogy, similarity and difference, and feminine and masculine and total English Semantic Test.
Table 2 Comparison between the two age groups regarding total and subtotal scores of the Arabic Semantic Test

\begin{tabular}{|c|c|c|c|c|c|}
\hline \multirow[t]{3}{*}{ Arabic Semantic Test } & \multicolumn{4}{|c|}{ Age groups } & \multirow[t]{3}{*}{$p$ value } \\
\hline & \multicolumn{2}{|c|}{$\begin{array}{l}\text { Group A (4 } \\
\text { to }<4.5)\end{array}$} & \multicolumn{2}{|c|}{$\begin{array}{l}\text { Group B (4.5 to } \\
<5)\end{array}$} & \\
\hline & Mean & $S D$ & Mean & $S D$ & \\
\hline Categorization & 30.74 & 1.29 & 31.15 & 1.06 & 0.141 \\
\hline Characteristic property & 21.00 & 1.41 & 21.12 & 1.60 & 0.721 \\
\hline Function & 19.15 & 0.91 & 19.24 & 0.83 & 0.640 \\
\hline Linguistic concepts & 16.77 & 1.80 & 16.91 & 1.51 & 0.709 \\
\hline Analogy & 23.49 & 1.14 & 23.64 & 0.74 & 0.518 \\
\hline Similarity and difference & 18.79 & 1.18 & 19.30 & 0.98 & $0.004^{*}$ \\
\hline Feminine and masculine & 10.91 & 1.23 & 11.09 & 1.13 & 0.517 \\
\hline Total & 130.85 & 3.55 & 131.45 & 4.85 & 0.365 \\
\hline
\end{tabular}

$S D$ standard deviation, bold: higher value, ${ }^{*}$ significant $p$ value $<0.05$

Table 3 Comparison between the two age groups regarding the total and subtotal scores of English Semantic Test

\begin{tabular}{|c|c|c|c|c|c|}
\hline \multirow[t]{3}{*}{ English Semantic Test } & \multicolumn{4}{|c|}{ Age groups } & \multirow{3}{*}{$p$ value } \\
\hline & \multicolumn{2}{|c|}{$\begin{array}{l}\text { Group A (4 to } \\
<4.5 \text { ) }\end{array}$} & \multicolumn{2}{|c|}{$\begin{array}{l}\text { Group B (4.5 } \\
\text { to }>5)\end{array}$} & \\
\hline & Mean & $S D$ & Mean & $S D$ & \\
\hline Categorization & 31.45 & 1.08 & 31.70 & 0.73 & 0.250 \\
\hline Characteristic property & 21.34 & 1.11 & 21.61 & 0.86 & 0.253 \\
\hline Function & 19.66 & 0.76 & 19.94 & 0.35 & 0.052 \\
\hline Linguistic concepts & 17.83 & 0.56 & 18.00 & 0.00 & 0.088 \\
\hline Analogy & 23.66 & 0.76 & 23.76 & 0.66 & 0.552 \\
\hline Similarity and difference & 19.28 & 0.97 & 19.45 & 0.90 & 0.409 \\
\hline Feminine and masculine & 11.57 & 0.83 & 11.82 & 0.58 & 0.150 \\
\hline Total & 132.56 & 3.85 & 135.96 & 4.95 & 0.376 \\
\hline
\end{tabular}

$S D$ standard deviation

Group A showed higher performance with a significant difference in the tasks of expressive and total categorization, total function, total linguistic concepts and its subtotal scores of color and shape, similarity and difference, and feminine and masculine in addition to total Semantic Test score in English than in Arabic language.

Group B children showed higher performance with a significant difference in tasks of expressive and total categorization, total function, total linguistic concepts and its subtotal scores of color, shape, and time, and total feminine and masculine in English more than in Arabic language, while similarity and difference score is significantly higher in Arabic more than English. 
Table 4 Comparison between the scores of Arabic and English Semantic Test in age group $A$

\begin{tabular}{|c|c|c|c|c|c|}
\hline \multirow[t]{2}{*}{ Group $A(4$ to $<4.5)$} & \multicolumn{2}{|c|}{$\begin{array}{l}\text { English } \\
\text { Semantic } \\
\text { Test }\end{array}$} & \multicolumn{2}{|c|}{$\begin{array}{l}\text { Arabic } \\
\text { Semantic } \\
\text { Test }\end{array}$} & \multirow[b]{2}{*}{$p$ value } \\
\hline & Mean & $S D$ & Mean & $S D$ & \\
\hline Receptive categorization & 12.00 & 0.00 & 11.91 & 0.41 & 0.160 \\
\hline Expressive categorization & 19.45 & 1.08 & 18.83 & 1.17 & $<0.001^{*}$ \\
\hline Total categorization & 31.45 & 1.08 & 30.74 & 1.29 & $<0.001^{*}$ \\
\hline $\begin{array}{l}\text { Receptive characteristic } \\
\text { property }\end{array}$ & 5.87 & 0.49 & 5.64 & 0.79 & 0.078 \\
\hline $\begin{array}{l}\text { Expressive characteristic } \\
\text { property }\end{array}$ & 15.47 & 0.97 & 15.36 & 0.97 & 0.574 \\
\hline Total characteristic property & 21.34 & 1.11 & 21.00 & 1.41 & 0.128 \\
\hline Total function & 19.66 & 0.76 & 19.15 & 0.91 & $<0.001^{*}$ \\
\hline Number & 2.00 & 0.00 & 1.85 & 0.51 & 0.051 \\
\hline Color & 4.00 & 0.00 & 3.62 & 0.77 & $0.001^{*}$ \\
\hline Shape & 3.87 & 0.49 & 3.55 & 0.83 & $0.002^{*}$ \\
\hline Time & 3.96 & 0.29 & 3.79 & 0.59 & 0.088 \\
\hline Total linguistic concepts & 17.83 & 0.56 & 16.77 & 1.80 & $<0.001^{*}$ \\
\hline Total analogy & 23.66 & 0.76 & 23.49 & 1.14 & 0.160 \\
\hline Total similarity and difference & 19.28 & 0.97 & 18.79 & 1.18 & $0.003^{*}$ \\
\hline Total feminine and masculine & 11.57 & 0.83 & 10.91 & 1.23 & $<0.001^{*}$ \\
\hline Total & 132.56 & 3.85 & 130.85 & 3.55 & $0.002^{*}$ \\
\hline
\end{tabular}

$S D$ standard deviation, bold: higher value, *significant $p$ value $<0.05$

Table 5 Comparison between the scores of Arabic and English Semantic Tests in age group $B$

\begin{tabular}{|c|c|c|c|c|c|}
\hline \multirow[t]{2}{*}{ Group B $(4.5$ to $<5)$} & \multicolumn{2}{|c|}{$\begin{array}{l}\text { English } \\
\text { Semantic } \\
\text { Test }\end{array}$} & \multicolumn{2}{|c|}{$\begin{array}{l}\text { Arabic } \\
\text { Semantic } \\
\text { Test }\end{array}$} & \multirow[b]{2}{*}{$p$ value } \\
\hline & Mean & $S D$ & Mean & $S D$ & \\
\hline Receptive categorization & 12.00 & 0.00 & 12.00 & 0.00 & --- \\
\hline Expressive categorization & 19.70 & 0.73 & 19.15 & 1.06 & $0.002^{*}$ \\
\hline Total categorization & 31.70 & 0.73 & 31.15 & 1.06 & $0.002^{*}$ \\
\hline $\begin{array}{l}\text { Receptive characteristic } \\
\text { property }\end{array}$ & 6.00 & 0.00 & 5.85 & 0.51 & 0.096 \\
\hline $\begin{array}{l}\text { Expressive characteristic } \\
\text { property }\end{array}$ & 15.61 & 0.86 & 15.27 & 1.42 & 0.190 \\
\hline Total characteristic property & 21.61 & 0.86 & 21.12 & 1.60 & 0.069 \\
\hline Total function & 19.94 & 0.35 & 19.24 & 0.83 & $<0.001^{*}$ \\
\hline Number & 2.00 & 0.00 & 1.94 & 0.35 & 0.325 \\
\hline Color & 4.00 & 0.00 & 3.64 & 0.78 & $0.002^{*}$ \\
\hline Shape & 4.00 & 0.00 & 3.64 & 0.78 & $0.002^{*}$ \\
\hline Time & 4.00 & 0.00 & 3.76 & 0.66 & $0.004^{*}$ \\
\hline Total linguistic concepts & 18.00 & 0.00 & 16.91 & 1.51 & $<0.001^{*}$ \\
\hline Total analogy & 23.76 & 0.66 & 23.64 & 0.74 & 0.402 \\
\hline Total similarity and difference & 19.36 & 0.90 & 19.40 & 0.96 & $0.004^{*}$ \\
\hline Total feminine and masculine & 11.82 & 0.58 & 11.09 & 1.13 & $<0.001^{*}$ \\
\hline Total & 135.96 & 4.95 & 131.45 & 4.85 & 0.364 \\
\hline
\end{tabular}

SD standard deviation, bold: higher value, ${ }^{*}$ significant $p$ value $<0.05$
Table 6 Comparison between the two age groups regarding the scores of the first Arabic Narration Test (the doll)

\begin{tabular}{|c|c|c|c|c|c|}
\hline \multirow[t]{3}{*}{ First Arabic story } & \multicolumn{4}{|c|}{ Age groups } & \multirow[t]{3}{*}{$p$ value } \\
\hline & \multicolumn{2}{|c|}{$\begin{array}{l}\text { Group A (4 to } \\
<4.5 \text { ) }\end{array}$} & \multicolumn{2}{|c|}{$\begin{array}{l}\text { Group B (4.5 to } \\
<5)\end{array}$} & \\
\hline & Mean & $S D$ & Mean & $S D$ & \\
\hline Story frame features & 9.60 & 1.47 & 10.42 & 1.09 & $0.007^{*}$ \\
\hline Events & 19.19 & 2.33 & 20.70 & 1.70 & $0.002^{*}$ \\
\hline Language structure & 5.72 & 0.68 & 5.88 & 0.48 & 0.265 \\
\hline Reference clarity & 2.91 & 0.28 & 2.91 & 0.29 & 0.929 \\
\hline Sentence structure & 1.70 & 0.72 & 1.82 & 0.58 & 0.446 \\
\hline Phrasal structure & 10.96 & 1.57 & 11.70 & 0.73 & $0.004^{*}$ \\
\hline Noun forms & 7.53 & 0.95 & 7.88 & 0.48 & 0.058 \\
\hline Verb forms & 7.62 & 0.90 & 7.82 & 0.58 & 0.262 \\
\hline Narrative productivity & 238.85 & 6.23 & 244.88 & 4.94 & $<0.001^{*}$ \\
\hline Total & 306.09 & 11.08 & 316.00 & 8.20 & $<0.001^{*}$ \\
\hline
\end{tabular}

Table 7 Comparison between the two age groups regarding the scores of first English Narration Test (the doll)

\begin{tabular}{|c|c|c|c|c|c|}
\hline \multirow[t]{3}{*}{ First English story } & \multicolumn{4}{|c|}{ Age groups } & \multirow[t]{3}{*}{$p$ value } \\
\hline & \multicolumn{2}{|c|}{$\begin{array}{l}\text { Group A (4 to } \\
<4.5 \text { ) }\end{array}$} & \multicolumn{2}{|c|}{$\begin{array}{l}\text { Group B (4.5 } \\
\text { to 5) }\end{array}$} & \\
\hline & Mean & $S D$ & Mean & $S D$ & \\
\hline Story structure features & 10.57 & 1.46 & 11.88 & 0.48 & $<0.001^{*}$ \\
\hline Events & 21.30 & 1.82 & 22.30 & 1.26 & $0.008^{*}$ \\
\hline Language structure & 5.51 & 0.86 & 6.00 & 0.00 & $0.002^{*}$ \\
\hline Reference clarity & 2.94 & 0.25 & 3.00 & 0.00 & 0.143 \\
\hline Sentence structure & 1.91 & 0.41 & 2.00 & 0.00 & 0.235 \\
\hline Phrasal structure & 11.00 & 1.68 & 11.94 & 0.35 & $0.002^{*}$ \\
\hline Noun forms & 7.83 & 0.56 & 7.94 & 0.35 & 0.325 \\
\hline Verb forms & 7.45 & 1.00 & 8.00 & 0.00 & $0.002^{*}$ \\
\hline Narrative productivity & 245.96 & 6.63 & 253.45 & 4.01 & $<0.001^{*}$ \\
\hline Total & 316.30 & 12.06 & 328.91 & 5.11 & $<0.001^{*}$ \\
\hline
\end{tabular}

SD standard deviation, bold: higher value, ${ }^{*}$ significant $p$ value $<0.05$

Bilingual English-Arabic Narration test (Tables 6, 7, 8, 9, 10, 11,12 , and 13)

$S D$ standard deviation, bold: higher value, "significant $p$ value $<0.05$

In the first Arabic story, age group B showed higher performance with a significant difference in the following items: story frame features, story events, phrasal structure, narrative productivity, and total score than group A.

Group B showed higher performance with a significant difference in the following items: story frame features, story events, language structure, phrasal structure, verb forms, narrative productivity, and total score than group A. 
Table 8 Comparison between the scores of Arabic and English Narration Test in the first story (the doll) in age group $A$

\begin{tabular}{|c|c|c|c|c|c|}
\hline \multirow[t]{2}{*}{ Group A (4 to < 4.5) } & \multicolumn{2}{|c|}{$\begin{array}{l}\text { First English } \\
\text { story }\end{array}$} & \multicolumn{2}{|c|}{$\begin{array}{l}\text { First Arabic } \\
\text { story }\end{array}$} & \multirow[t]{2}{*}{$p$ value } \\
\hline & Mean & $S D$ & Mean & $S D$ & \\
\hline Story structure features & 10.57 & 1.46 & 9.60 & 1.47 & $<0.001^{*}$ \\
\hline Events & 21.30 & 1.82 & 19.19 & 2.33 & $0.008^{*}$ \\
\hline Language structure & 5.51 & 0.86 & 5.72 & 0.68 & $0.002^{*}$ \\
\hline Reference clarity & 2.94 & 0.25 & 2.91 & 0.28 & 0.143 \\
\hline Sentence structure & 1.91 & 0.41 & 1.70 & 0.72 & 0.235 \\
\hline Phrasal structure & 11.00 & 1.68 & 10.96 & 1.57 & $0.002^{*}$ \\
\hline Noun forms & 7.83 & 0.56 & 7.53 & 0.95 & 0.325 \\
\hline Verb forms & 7.45 & 1.00 & 7.62 & 0.90 & $0.002^{*}$ \\
\hline Narrative productivity & 245.96 & 6.63 & 238.85 & 6.23 & $<0.001^{*}$ \\
\hline Total & 316.30 & 12.06 & 306.09 & 11.08 & $<0.001^{*}$ \\
\hline
\end{tabular}

$S D$ standard deviation, bold: higher value, *significant $p$ value $<0.05$

Table 9 Comparison between the scores of Arabic and English Narration Tests in the first story test (the doll) in age group $B$

\begin{tabular}{|c|c|c|c|c|c|}
\hline \multirow[t]{2}{*}{ Group B $(4.5$ to <5) } & \multicolumn{2}{|c|}{$\begin{array}{l}\text { First English } \\
\text { story }\end{array}$} & \multicolumn{2}{|c|}{$\begin{array}{l}\text { First Arabic } \\
\text { story }\end{array}$} & \multirow[t]{2}{*}{$p$ value } \\
\hline & Mean & $S D$ & Mean & $S D$ & \\
\hline Story frame features & 11.88 & 0.48 & 10.42 & 1.09 & $0.002^{*}$ \\
\hline Events & 22.30 & 1.26 & 20.70 & 1.70 & 0.254 \\
\hline Language structure & 6.00 & 0.00 & 5.88 & 0.48 & 0.265 \\
\hline Reference clarity & 3.00 & 0.00 & 2.91 & 0.29 & 0.929 \\
\hline Sentence structure & 2.00 & 0.00 & 1.82 & 0.58 & 0.446 \\
\hline Phrasal structure & 11.94 & 0.35 & 11.70 & 0.73 & $0.014^{*}$ \\
\hline Noun forms & 7.94 & 0.35 & 7.88 & 0.48 & 0.058 \\
\hline Verb forms & 8.00 & 0.00 & 7.82 & 0.58 & 0.262 \\
\hline Narrative productivity & 253.45 & 4.01 & 244.88 & 4.94 & $<0.001^{*}$ \\
\hline Total & 328.91 & 5.11 & 316.00 & 8.20 & $<0.001^{*}$ \\
\hline
\end{tabular}

SD standard deviation, bold: higher value, ${ }^{*}$ significant $p$ value $<0.05$

Table 10 Comparison between the two age groups regarding the scores of second Arabic Narration Test (the boy and his grandpa)

\begin{tabular}{|c|c|c|c|c|c|}
\hline \multirow[t]{3}{*}{ Second Arabic story } & \multicolumn{4}{|c|}{ Age groups } & \multirow{3}{*}{$p$ value } \\
\hline & \multicolumn{2}{|c|}{$\begin{array}{l}\text { Group A (4 to } \\
<4.5)\end{array}$} & \multicolumn{2}{|c|}{$\begin{array}{l}\text { Group B (4.5 to } \\
<5)\end{array}$} & \\
\hline & Mean & $S D$ & Mean & $S D$ & \\
\hline Sentence repetition & 13.89 & 1.78 & 14.79 & 1.19 & $0.014^{*}$ \\
\hline Code switch & 3.04 & 0.92 & 3.88 & 0.99 & $<0.001^{*}$ \\
\hline Total & 16.04 & 2.56 & 17.41 & 2.63 & $<0.001^{*}$ \\
\hline
\end{tabular}

SD standard deviation, bold: higher value, ${ }^{*}$ significant $p$ value $<0.05$
Table 11 Comparison between the two age groups regarding the scores of second English Narration Test (the boy and his grandpa)

\begin{tabular}{lllllll}
\hline Second English story & \multicolumn{2}{l}{ Age groups } & \\
\cline { 2 - 3 } & $\begin{array}{l}\text { Group A (4 to } \\
<\mathbf{4 . 5})\end{array}$ & & $\begin{array}{l}\text { Group B (4.5 } \\
\text { to 5) }\end{array}$ & p value \\
\cline { 2 - 3 } & Mean & $S D$ & & Mean & SD & \\
\hline Sentence repetition & 14.86 & 0.78 & $\mathbf{1 4 . 8 9}$ & 0.91 & $<0.001^{* *}$ \\
Total & 16.48 & 0.48 & $\mathbf{1 7 . 6 4}$ & 0.52 & $<0.001^{*}$ \\
\hline
\end{tabular}

SD standard deviation, bold: higher value, ${ }^{*}$ significant $p$ value $<0.05$

Table 12 Comparison between the scores of Arabic and English Narration Tests in the second story (the boy and his grandpa) in age group $A$

\begin{tabular}{llllllll}
\hline Group A (4 to < 4.5) & \multicolumn{2}{l}{ English } & & & Arabic & & \multirow{2}{*}{$\boldsymbol{p}$ value } \\
\cline { 2 - 3 } & Mean & SD & & Mean & SD & \\
\hline Sentence repetition & $\mathbf{1 4 . 8 6}$ & 1.78 & & 13.89 & 1.78 & $<0.001^{*}$ \\
Code switch & 2.00 & 0.00 & $\mathbf{2 . 2 3}$ & 0.92 & $<0.001^{*}$ \\
Total & 16.04 & 1.54 & $\mathbf{1 6 . 1 4}$ & 2.56 & $<0.001^{*}$ \\
\hline
\end{tabular}

SD standard deviation, bold: higher value, ${ }^{*}$ significant $p$ value $<0.05$

Table 13 Comparison between the scores of Arabic and English Narration Tests in the second story (the boy and his grandpa) test in age group $B$

\begin{tabular}{llllllll}
\hline Group B (4.5 to < 5) & \multicolumn{2}{l}{ English } & & & Arabic & & p value \\
\cline { 2 - 3 } & Mean & SD & & Mean & SD & \\
\hline Sentence repetition & $\mathbf{1 4 . 8 9}$ & 0.91 & & 14.79 & 1.19 & $<0.001^{*}$ \\
Code switch & 2.00 & 0.00 & $\mathbf{3 . 8 8}$ & 0.99 & $0.002^{*}$ \\
Total & $\mathbf{1 7 . 6 4}$ & 0.52 & 17.41 & 2.56 & $<0.001^{*}$ \\
\hline
\end{tabular}

Group A children showed higher performance with a significant difference in English than Arabic in the following items: story frame features, events, phrasal structure, narrative productivity, and total scores of the first story, while they showed higher performance with a significant difference in the first Arabic story regarding language structure and verb forms than first English story.

Group B children showed higher performance with a significant difference in the following items: story frame features, phrasal structure, narrative productivity, and total score of the first story in English than in Arabic.

In the second Arabic story, age group B shows higher performance with a significant difference in sentence repetition, code switch, and total score than group $\mathrm{A}$. 
Group B showed higher performance with a significant difference in sentence repetition and total score than group A, while scores of code switch showed no significant difference between the two age groups and had constant scores.

Group A children showed higher performance with a significant difference regarding sentence repetition in English than Arabic while they showed more use of code switch and higher total score in Arabic than English.

$S D$ standard deviation, bold: higher value, *significant $p$ value $<0.05$

Group B children showed higher performance in English than Arabic regarding sentence repetition score and total score, while they showed more use of code switch in Arabic than English.

\section{Reliability (Table 14)}

Cronbach's alpha test showed acceptable reliability of Arabic and English Semantic and Narration Tests

\section{Discussion}

\section{Bilingual English-Arabic Test (BEAT)}

Assessment of the components of the two languages is considered the standard for the assessment of bilingual children [15]. This provides appropriate information about bilingual normative data to be able to assess bilingual children accurately and not to be misdiagnosed as having language impairment.

One of the challenges in bilingual testing is the use of translation of the test, but it is generally agreed that translated tests do not have the same psychometric properties as the original test. Translated tests from one language to another may emphasize forms that are not clinically sensitive in another language [16]. Also, bilingual children may demonstrate their knowledge in different ways. This means it is necessary to build tests around tasks that are age appropriate to children under the study using familiar and common items in their environment.

The BEAT semantic assessment was designed to be used with bilingual Egyptian children who speak both Arabic and English. Important considerations were taken for the development of language tests for the cultural and linguistic appropriateness as well as the variability in children's first and second language experiences. So, the current assessment was designed as an attempt aiming at evaluating the characteristics in bilingual languages that may help clinicians make accurate and reliable diagnostic decisions.

Table 14 Reliability of test

\begin{tabular}{ll}
\hline Bilingual Arabic English Test & Cronbach's alpha \\
\hline Arabic Semantic and Narration Tests & 0.847 \\
English Semantic and Narration Tests & 0.727 \\
\hline
\end{tabular}

\section{Semantic section}

Semantic tasks are very important for the assessment of bilinguals because it is often measured in levels of vocabulary knowledge, so it is vital for language development because children who understand content can comprehend language more fully and have a greater variety of ways to express themselves. It is also easily translated and takes little time to administer [17]. This semantic test included tasks that required deep knowledge of words such as categorical and functional information, variations of words, and the sociolinguistic contexts of word usage [18].

In the current study, many semantic tasks were administered with preschool-aged bilingual children. Each of these tasks was able to gain insights into the types of changes in semantic structures as well as the factors that drive these changes such as the categorization task which was proved to examine how categories and their examples are organized in both languages and how semantic information is represented in bilingual speakers as they are able to process categorical word examples of one language faster by filtering out unrelated meanings to the category mentioned in other languages.

\section{BEAT Semantic Arabic Test (Table 2)}

Table 2 results reveal that the similarity and difference task developed in Arabic as early as 4 years old apart from the similarity and difference task that continues to develop after 4.5 years. This result is in agreement with that of a study by McLeod [19] who showed that 5 -year-old children develop the ability to talk about things, describe them, and tell the difference between them due to linguistic skills development and cognitive development which allow recognition of similarities or differences between objects through recalling previous information about these objects and mental categorization according to similarity or difference between them with their vocabulary will continue to grow while growing of their semantic capacity [20].

\section{BEAT Semantic English Test (Table 3)}

Table 3 results go with a study done on comparing English language development on different age groups (4 and 5 years old) which revealed equal development due to the cognitive and intellectual nature of language development which undergoes at a similar rate and timing [21].

This is in agreement with a study done by Hoff [22] who dedicated equal English skill levels in 4- and 5 -year-old children as they hear a heritage language at home since birth and their first systematic exposure to 
English begins with preschool or kindergarten. Therefore, the development of English skills in these ages showed a limited difference.

\section{Arabic versus English BEAT Semantic test in bilingual age group A (Table 4)}

Table 4 results are probably due to that with age increasing, there is an increase in vocabulary depth that allows acquiring of more vocabulary and categorizes them mentally in semantic categories and the ability to separate the two languages from each other.

At the individual level, a majority of the children showed increased accuracy for the English tasks more than other languages and that is in agreement with Sheng [23]. In addition, Oller and Cobo-Lewis [24] reported rapid gains in the number of entries in their English vocabulary (breadth of vocabulary) during preschool and school-age years more than native language in Spanish-English bilingual children.

\section{Arabic versus English BEAT Semantic test in bilingual age group B (Table 5)}

Table 5 shows that children had higher performance in English more than Arabic test in most of the items apart from similarity and difference task which is the only item that is higher in Arabic than English. That indicates that these skills are more developed in English than Arabic except for similarity and difference item which develops at a different rate as bilingual children may show relative strength in a given domain in one language while demonstrating strength in a different domain in the other language. Mixed dominance has been documented for a significant proportion of bilingual children [25] and is not considered an indicator of impairment. Children both with and without language impairment may present with "mixed dominance" [26].

As noticed during testing, bilingual children may not know the same words in each language and this depends on their experiences and language exposure. Gaps in vocabulary can be problematic for school-age children who must use specific words in academic tasks [27]. However, bilinguals may use their knowledge of vocabulary in one language as a bootstrap to facilitate word learning in the other [28].

However, older age group children showed equal performance in both languages regarding receptive categorization, receptive, expressive and total characteristic property, subtotals of linguistic concepts such as adjective and number, total analogy, and total semantic test score.

\section{Narration section}

The narrative task has been shown to be a clinically sensitive measure of language abilities of bilingual children [29] as certain measures of narrative ability have been reliably used to distinguish between children with and without language impairment [30]. According to Oakhill [31], the ability to tell a story links oral language skill to literacy, since it requires children to plan and produce contextualized and cohesive discourse.

Oral narratives provide a rich source of data about a child's language use in a relatively natural context. So, narrative analysis allows clinicians to assess multiple linguistic features in context including macrostructure, e.g., story frame structure, and microstructure features, e.g., noun form, verb form, phrasal structure, and finally specific bilingual strategies as code switch [32].

Based on the literature and given the importance of narrative skills, they were included in the current assessment through the use of two stories.

\section{First story (retelling): The doll}

Macrostructure Narrative skills have been used to assess both the global skill of reconstructing a story as well as a range of different sub-skills of children's language. Regarding sub-skills, narrative skills are a good indicator of linguistic complexity, often requiring the use of subordinate clauses (clause, typically introduced by a conjunction, that forms part of and is dependent on a main clause) to specify the cause or purpose of a particular action alongside the description of the action itself [33]. In addition, narratives can also give an indication of children's discourse skills, in particular the introduction of referents, topic maintenance, location of an action in time, and use of connectives. That is known as macrostructure [34].

Macrostructure takes into account a child's ability to convey a thematically coherent story, relying on skills, such as conceptualizing and planning sequences of events, and making inferences about characters' motivations [35].

Story frame structure The macrostructure analysis focuses on the concept of the story frame structure, which involves the presence of the setting (time and place) and the episode structure [36], with its core components of the goal of the protagonist (characters), the attempt to reach the goal, and the outcome of the actions [37]. The children in the first story listen carefully to the assessor while telling the story as they were asked to retell it again sticking as much as they can to the given words, meaning, and content of the story. 
Tables 6 and 7 show that older age group children were more proficient in story frame structure in both languages more than younger age group. This was confirmed by a study done by Hutson [38] on English and Hebraic speakers which found that the onset of narrative abilities seems to occur at 3 years of age. Between 3 and 5 years of age, the children progress from object description and temporal sequences of relevant actions to the solution of problems. Some studies have suggested that the development of narrative abilities reaches its highest level of complexity around age 4.5 years.

Also, Tables 8 and 9 show higher performance in the English language than Arabic regarding the total story frame structure and phrasal structure. This difference may be due to different rates of development of aspects of oral narratives in both languages in the younger age group and in the older age group, Arabic is less developed. The most probable explanation was that the older age group are having higher cognitive abilities, so they develop using code switching more professionally than younger age with more exposure to the English language at school and expansion of their English vocabulary and more exposure to English songs, stories, and use at home which was a way done by the parents to assist and encourage the development of the second language.

Similarly, Ordóñez [39] stated that bilinguals in the monolingual environment (the environment where the people only speak the native language), in which their bilingual education takes place, had limitations in their native language (Spanish) stories. These limitations are probably linguistically in nature, and that may have prevented the bilinguals from demonstrating the whole range of cognitive abilities they may be able to display when telling a linguistically complex story. The limitations to the native language were probably attributed to that bilingualism broadens students' access to advanced education in an increasingly globalized world. Thus, there is more desire to use and learn the English language more than the native language, so as to allow wider access to early English semi-immersion, as the presumed best way to achieve early bilingualism.

Tables 8 and 9 show that English story macrostructure performance was better than Arabic in both age groups although language structure and verb form (as microstructure linguistic skills) was better in Arabic story than English story especially in the younger age group due to the more use of the verb form and language structure items (such as intensifiers) at home in the Arabic language by the parents while giving orders or narrating a situation or a story to younger than older age children. This also showed the variation in development in micro- and macrostructure in both languages. This was in agreement with Rodina [40] who revealed that bilinguals may lack some language-specific skills. His or her general narrative abilities reflected in macrostructure categories will grow in both languages interdependently and might enhance each other's growth. Such a carry-over effect is possible due to knowledge transfer between the two languages.

Narrative productivity Tables 6 and 7 show a significant difference between the two age groups regarding the narrative productivity items where the older age group showed higher performance than the younger age group. This reflects the age development and the increase in MLU (mean length of utterance/average sentence length) as sentence complexity and length increase gradually with age. This was shown in the language development process in which MLU in 4 years is 4.6 words, 4.5 years is 5 words, and 5 years is 5.6 words.

Tables 8 and 9 show that narrative productivity was more developed in English than in the Arabic language. This may be due to the more developed English language leading to an increase in mean length of utterance which reflects the increase in sentence length and complexity. In addition, the study revealed that the type token ratio was higher in English than in the Arabic language which reveals higher variation in the English vocabulary used and the increase in the complexity of the children's narrative English language.

This could be explained by various sources of exposure to the English language in the form of more watching English cartoons, listening to English songs and stories than in Arabic, in addition to the school attitude in encouraging the use of the second language in the classroom. All these factors allow the children to be more exposed and proficient in the second language than the native language.

Similarly, a study made by Merrikhi [41] on Arabic and English languages showed that bilingual children tend to exhibit unequal performance in their two languages. Bilingual utterance length (MLU) was much higher in the second language than the first language. This may be interpreted as increase sentence complexity and variability in the vocabulary of the second language including grammar of the English language in which bilinguals outperformed on more than native language

Microstructure Microstructure is more language-specific than macrostructure because it depends on the proficiency of the bilingual children's language, such as syntactic knowledge [27]. 
Tables 6 and 7 show that older age children showed higher performance in language structure, phrasal structure, and verb forms than the younger age group in both languages. This may be due to the development of language complexity and sentence structure with age gaining more linguistic skills such as morpho-syntax as a reflection of cognitive development.

Similarly, 4.5- to 5-year-old children will keep getting better at storytelling as at this age, and they will speak in increasingly complex sentences by joining small sentences together using words like "and" or "because." They also will be able to use long sentences of up to nine words. They will develop the ability to talk about things that have happened in the past or will happen in the future as well as irregular plurals like "mice" and pronouns like "him," "his," and "her" [42].

The microstructure measures of bilingual narratives do not only reflect the child's age, but also the actual lexical and morpho-syntactic development of each of their languages. At a given moment, microstructure measures may remain language-specific and showcase differences in the narrative abilities between the bilingual's languages [43].

Tables 8 and 9 show that both age groups showed higher performance in English more than Arabic in phrasal structure (which consists of adjective, adverb, negation, preposition phrase, interrogative phrase, and intention phrase). This may be due to easier phrasal structure components in the English language than in Arabic which allows their early development in the English language.

This was similar to the kindergarten English retelling that was observed to contain more microstructure elements such as adjective, adverbs, and negation than the retells in the native language (Spanish) in the study of Squires [44]. In a study done on English/Spanish narrative skill development in the USA by Uccelli and Páez [14], it was found that bilingual children produced better performances in English as a second language than in Spanish (native language), with larger differences on language elements (microstructure) than on story features (macrostructure); this was due to different rates of development of language skills in one of both languages.

However, Tables 8 and 9 show that both age groups develop some narration items equally such as temporality and connectives, reference clarity, sentence structure, and noun form.

So, the current study revealed different developmental rates in macrostructure and microstructure between both languages, as macrostructure (story frame structure, events, and narrative productivity) performance by bilingual children under the study was better in English than in the Arabic language. In microstructure, the phrasal structure item (which is a type of rule used to describe a given language's syntax and are closely associated with the early stages of transformational grammar such as adjective, adverb, negation, and interrogative phrase) was better in English than in the Arabic language, while language structure (which is defined as words and sentences have parts that combine in patterns, exhibiting the grammar of the language such as specificity, temporality, connectives, and reference clarity) and verb form were better in Arabic than the English language.

\section{Second story (sentence repetition/mixing)}

This story was used in order to elicit strategies used by bilingual children such as code switching and mixing.

Sentence repetition The second story is used to evaluate the children's ability to repeat sentences presented to them in an interactive way so as to detect any characteristic errors in this area. Errors of sentence repetition task were probably expected to be either due to the effect of bilingualism on language in the form of mixing between both languages and cross-language transfer or memory errors. The memory errors can be presented in the form of omission or deletion of one or more of the presented words in the administered sentences or in the form of commission (substitution of the words by other words of the same language due to memory problem). During analysis of the sentence repetition task, most of the errors were found to be linguistic errors in the form of mixing between the two languages (from Arabic to English was more common than from English to Arabic language and errors due to cross-language transfer as the effect of English on Arabic was higher than that of Arabic to English).

Therefore, the children's responses were scored on a scale from 0 to 4 according to the number of errors committed by the children under the study. The reason beyond using the scale of score from 0 to 4 instead of 0 , 1 score was that the sentence repetition task changes the effect of the testing by measuring the number of errors in a sentence. This was the most successful method for differentiating between language-impaired children and bilingual children.

Tables 10 and 11 show that the older age group performed higher in both languages than the younger age group in the sentence repetition task. This may be due to the development of both language abilities in which 
these language skills develop with age. This goes with Klem [45] who revealed that sentence repetition appears to be a valuable tool for language assessment because it draws upon a wide range of language skills as sentence repetition offer a window into grammatical skills, phonology, morpho-syntax, and semantics in order to be able to repeat the sentences by processing and reconstructing their meaning. This can only happen if the participant has already acquired and developed the grammatical structures and other narrative skills [46].

Tables 12 and 13 show that less errors were detected in the sentence repetition task in English than in Arabic. This was secondary to the bilingual children's use of code switching more from Arabic to the English language, and this was to fill the linguistic gap produced from a deficient vocabulary of the native language. Also, bilingual children tend to use cross-language transfer which showed more influence of the English language on the native language as linguistic transfer depends on the levels of proficiency in the two languages. This confirmed that sentence repetition errors in bilingual children under the study were more linguistic errors, in Arabic than the English language rather than memory errors.

A study done by Muñoz [47] revealed that bilingual children have intact cognitive skills such as memory and attention as they are proficient on the more difficult sequential condition and showed more efficient and faster recalling and monitoring systems.

Code switch Table 10 shows that older age group children used code switching from the Arabic language into English more often than younger age group children. This is explained by the ability of bilingual children to keep intact understandable conversation by filling the gap, shifting in using vocabulary between the two languages. One of the most frequent explanations of why bilinguals use code switch is that they do it to compensate for lack of language proficiency because they do not know either language completely.

Researchers in the second language acquisition tradition have also focused on the age effect on bilingual strategies [48] as the older the children's age, the larger the numbers of semantic responses and fewer errors; this was explained by Sheng [49] who showed that the amount of language experience would affect the depth of semantic knowledge, resulting in an asymmetry in semantic depth between a bilingual's more and less frequently used languages.

However, Table 11 shows that there was no significant difference between the two age groups when using code switch from the English to Arabic language. This is probably due to that there is less common switching from the English language to Arabic which might be due to more development of second language vocabulary and acquisition of a variety of vocabulary which are used while speaking in the second language.

\section{Reliability: Table 14}

Test reliability refers to the consistency of a measure or test. A test will be reliable when it gives the same repeated result under the same conditions. It is measured by Cronbach's alpha test which is how closely related a set of items are as a group. Alpha coefficients above 0.9 are considered excellent. This study showed acceptable reliability of the Arabic and English Semantic and Narration Tests $(0.847,0.727$ respectively) as evidenced by the significant correlation between the assessment items and the subitems within the same item. This in turn reflected the internal consistency of the designed tool. This was similar to Peña [6] in Bilingual English-Spanish Assessment (BESA) which revealed reliability of all data was just under 0.8 and was in the acceptable range.

\section{Test validity}

The BEAT designed in this study was not validated against other tests as the key reason for this is that there is no testing for bilingual children available in the Egyptian community.

\section{Content validity}

The items used in this study were selected carefully from the literature. All Arabic language tests available for the Egyptian children addressing the age group under the study were reviewed. The items selected had also been studied extensively by several research groups (APPLE TOOL by Osman [8], REAL Scale by Osman [9], Pls 4 by Abu Haseeba et al. [10], The Arabic language test revised by Rifaie et al [11], and Assessment of Narrative Skills in Preschool Children by Kamal et al. [12]).

\section{Study limitations}

The study is a trial to collect normative data to understand the profile of language development in bilingual children in this young age. However, the study showed some limitations as applying the test on a larger scale and a wider age range is warranted. Thorough evaluation of all language aspects is thought to be helpful for more understanding of the bilingual children development. Other types of reliability such as interrater and intrarater reliability or test and retest reliability are needed to ensure the internal consistency for this designed study. 


\section{Conclusions}

This was demonstrated through the results of the study which showed that older age group children had better semantic (Arabic similarity and difference item) and narration skills of macrostructure (story frame structure, events, and narrative productivity) and microstructure (language structure, phrasal structure, verb and noun form) in both languages. Semantic developed in both languages at the same rate except for similarity and difference items which were found to develop at different rates (earlier in Arabic than the English language). Meanwhile, in the narrative assessment of the current study, children's performance in macrostructure items (such as story frame and narrative productivity) especially in the older age group was better in the English language while children of the younger age group develop microstructure items (such as language structure and verb form) earlier in the Arabic language. Also, the sentence repetition task in both age groups is better in English than in the Arabic language because of less errors of code switch. Code switching was more obvious from Arabic to English and in the older age group more than the young age group. The Bilingual EnglishArabic Test (BEAT) showed acceptable reliability.

\section{List of table legends}

Subtests: The language items tested in bilingual children

Items in each subtest: The number of items in each subtest

Scoring of each subtest: The total scoring for each subtest

Arabic Semantic Test: Semantic section in the Arabic language of Bilingual English-Arabic Test

English Semantic Test: Semantic section in the English language of Bilingual English-Arabic Test

Arabic narrative test: Narrative section in the Arabic language of Bilingual English-Arabic Test

English narrative test: Narrative section in the English language of Bilingual English-Arabic Test

Group A: Children with age group from 4 years old to 4 years and 6 months

Group B: Children with age group from 4 years and 6 months to 4 years and 11 months

Categorization: Identifying an item from a certain category; then labeling the category of certain items

Characteristic property: Identifying the correct item according to a description given, then describing the object and its attributes

Function: Identifying and labeling an object function provided by the picture

Linguistic concept: Identifying some linguistic concepts like features, time, color, number, function, and shape
Similarities and differences: Giving one similarity and one difference between two objects provided by pictures

Feminine and masculine: Identifying and using subjective, objective pronouns, and possession

Analogy: Labeling the relationship between object pairs provided by pictures

Story frame features: Narrative elements used to evaluate the child's ability to put proper outline for story features starting by giving an introduction, the orientation of character, 10 actions, 1 setting, 1 time, and story ending (coda)

Events: Narrating all the events in a given story

Language structure: Consists of "Specificity" that means words that give more details, such as intensifiers, and words referring to physical and emotional state; "temporality" which means subjective progression of timeeg the word "after"; connective which means using certain words connecting sentences to give appropriate meaning such as "and," "and so," and "therefore"; and reference clarity which means retelling the content to be easy to be understood with appropriate grammatical properties such as telling presupposed reference using definite article and noun

Sentence structure: Narrating a compound sentence

Phrasal structure: Contains adjective, adverb, negation, and interrogative phrase

Noun forms: Consists of regular and irregular plurals, possession, colors, and conjoined noun

Verb forms: Present tense, past tense, future tense, auxiliary verb (in English)

Narrative productivity: Consists of the total number of words, the mean length of utterances, and the ratio of number of different words spoken over total number of words (type token ratio)

Sentence repetition: Sentences repeated by the children within the second story

Code switching: Switching between both languages done by bilingual children within the stories

\section{Supplementary Information}

The online version contains supplementary material available at https://doi. org/10.1186/s43163-022-00209-x.

Additional file 1. Bilingual English-Arabic Test (BEAT)

\section{Acknowledgements}

Not applicable

Authors' contributions

SM is the corresponding author who contacted the journal, implemented the assessment, applied on the cases, analyzed and interpreted the children's data, and wrote the manuscript. AF author thought for the idea, and AA and DM authors shared in the design of the idea and the assessment protocol and 
revision and editing of the manuscript and assessment. The authors read and approved the final manuscript.

\section{Funding}

This research did not receive any specific grant for funding agencies in the public, commercial, or not-for-profit sectors.

\section{Availability of data and materials}

The datasets generated and analyzed during the current study are not publicly available due to sharing of the data is confined to the authors of the study.

\section{Declarations}

\section{Ethics approval and consent to participate}

The present study was with ethical number 160-2018. This study was approved by the Medical Research Ethics Committee of the phoniatric department and Ethical Committee of AlKasrAIEiny University on 5 December 2018. The consent was taken from the Directorate of Education in Cairo before starting the study. Some schools in Greater Cairo Educational Region were chosen according to school approval to implement the protocol of assessment on their children. A security approval to these schools which were selected for this study was taken. An informed written consent was taken from all the parents/guardians of all participants to apply this language test in which it includes the methods of applying the test and privacy control, and no hazards have resulted from the test.

\section{Consent for publication}

Not applicable

\section{Competing interests}

The authors declare that they have no competing interests.

Received: 14 November 2021 Accepted: 4 January 2022 Published online: 23 February 2022

\section{References}

1. Hamers JF, Blanc M, Blanc MH, Hamers JF (2000) Bilinguality and bilingualism. Cambridge University Press

2. Grosjean F (2010) Bilingual. Harvard University Press

3. Ong F, Nguyen SD (2009) Preschool English learners, principles and practices to promote language, literacy and learning. A resource guide (second edition) Child Development Division. California Department of Education

4. Meisel J (1989) Early differentiation of languages in bilingual children. In: Hyltenstam K, Obler L (eds) Bilingualism across the lifespan. Aspects of acquisition, maturity and loss. Cambridge University Press, Cambridge

5. Peña ED, Bedore LM, Iglesias A, Gutiérrez-Clellen VF, Goldstein BA (2008) Bilingual English Spanish Oral Screener-Experimental version (BESOS). Int J Bilingual Educ Bilingualism. 11(1):1-29

6. Peña ED, Gutiérrez-Clellen VF, Iglesias A, Goldstein BA, Bedore LM (2018) Bilingual English Spanish Assessment (BESA). San Rafael, Clinical Publications. Brookes, Baltimore, MD 1-800-638-3775

7. Gutiérrez-Clellen VF, Pena E (2001) Dynamic assessment of diverse children. Lang Speech Hearing Serv Schools 32:212-224

8. Osman DA (2014) Receptive Expressive Arabic Language Scale (REAL Scale). Print House, Cairo, Egypt

9. Osman DA (2017) A Proficiency Preschool Language Evaluation (APPLE TOOL)- version 2: assessment tool for Arabic speaking children from birth through 4 years, 11 month ( $<5$ years old ). Print House office, Cairo, Egypt

10. Abu Haseeba A, El Sady S, Elshobary A, Gamal N, Ibrahim M, Abd ElAzeem A (2011) Standardization, translation and modification of the preschool language scale - 4MD Thesis submitted to phoniatric unit. Ain Shams University, Cairo

11. Rifaie N, Hamza TMAW, Elfiky YH (2021) Validity and reliability of the revised Arabic language test for 2-4-year-old children: cross-sectional study. Egypt J Otolaryngol 37:26
12. Kamal IA, Desoky H, Shohdi SS, Safwat RF (2012) Assessment of narrative skills in preschool children. Published M.Sc. thesis of phoniatrics presented by Kamal I A, Faculty of medicine, Cairo University

13. Maged S, Aziz A A, Osman D A, Abdelhady A A (2020): A preliminary evaluation of some language aspects in normal bilingual Egyptian kindergarten children, unpublished thesis for fulfillment of master degree of phoniatric, Phoniatric department, Faculty of Medicine, Cairo University.

14. Uccelli P, Páez M (2007) Narrative and vocabulary development of bilingual children from kinder-garten to first grade, developmental changes and associations among English and Spanish skills. Lang Speech Hearing Serv Schools J 38(3):225-236

15. Kohnert KJ (2010) Bilingual children with primary language impairment: issues, evidence and implications for clinical actions. J Commun Disord 43:456-473

16. Arnold BR, Matus YE (2000) Test translation and cultural equivalence methodologies for use with diverse populations. In: Cuellar I, Paniagua FA (eds) Handbook of multicultural mental health. Academic Press, San Diego, CA

17. Betz SK, Hoff E, Sullivan SF (2013) Factors influencing the selection of standardized tests for the diagnosis of specific language impairment. Lang Speech Hearing Serv Schools 44(2):133-146

18. McCardle P, Leung CYY (2006) English language learners, development and intervention - an introduction. Top Lang Disord 26:302-304

19. McLeod S, Crowe K (2018) Children's consonant acquisition in 27 languages. A cross-linguistic review. Am J Speech Lang Pathol 27:1546-1571

20. MayberryRI (1993) First-language acquisition after childhood differs from second-language acquisition: the case of American Sign Language. J Speech Lang Hearing Res 36(6):1258-1270

21. Muluk NB, Bayoğlu B, Anlar B (2013) Language development and affecting factors in 3-to 6-year-old childre. Eur Arch Otorhinolaryngol 271(5):871-878

22. Hoff E (2013) Interpreting the early language trajectories of children from low SES and language minority homes: implications for closing achievement gaps. Dev Psychol 49:4-14

23. Sheng L, Peña ED, Bedore LM, Fiestas CE (2012) Semantic deficits in Spanish-English bilingual children with language impairment. J Speech Lang Hearing Res 55:1-15

24. Oller DK, Pearson B, Cobo-Lewis A (2007) Profile effects in early bilingual language and literacy acquisition. Appl Psycholinguist 28:198-229

25. Bedore L, Peña E, Summer C (2012) The measure matters: language dominance profiles across measures in Spanish-English bilingual children. Bilingualism Lang Cognition 10(17):36-67

26. Paradis J, Crago M (2003) Tense and temporality: a comparison between children learning a second language and children with SLI. J Speech Lang Hearing Res 43:834-847

27. Carol A, Hulle V et al (2004) Genetic, environmental, and gender effects on individual differences in toddler expressive language. J Speech Lang Hearing Res 47:904-912

28. McGregor KK, Oleson J, Bahnsen A, Duff D (2013) Children with developmental language impairment have vocabulary deficits characterized by limited breadth and depth. Int J Lang Commun Disord 48(3):307-319

29. Bedore LM (2010) Language sample measures and language ability in Spanish English bilingual kindergarteners, Department of Communication Sciences and Disorders, The University of Texas at Austin. J Commun Disord 43(6):498-510

30. Iluz-Cohen P, Walters J (2012) Telling stories in two languages: narratives of bilingual preschool children with typical and impaired language. Bilingualism Lang Cognition 15:58-74

31. Oakhill J, Cain K (2007) Issues of causality in children's reading comprehension. In: McNamara D (ed) Reading comprehension strategies: theories, interventions, and technologies, vol 17, pp 47-72

32. Heilmann J, Miller JF, Nockerts A (2010) Sensitivity of narrative organization measures using narrative retells produced by young school-age children. Lang Test 27:603-626

33. Gutiérrez-Clellen VF, Kreiter J (2003) Understanding child bilingual acquisition using parent and teacher reports. Appl Psycholinguist 24:267-288

34. Hickmann M (2003) Children's discourse: person, space and time across languages. Cambridge, UK: Cambridge University Press 66(9): 314-396.

35. Montanari S (2004) The development of narrative competence in the L1 and L2 of Spanish-English bilingual children. Int J Biling 8:449-497 
36. Stein NL, Glenn CG (1979) An analysis of story comprehension in elementary school children. In: Freedle R (ed) New directions in discourse processing, vol 53. Albex, Hillsdale, pp 648-669

37. Gagarina N, Klop D, Kunnari S, Tantele K, Valimaa T (2016) Assessment of narrative abilities in bilingual children. In: Armon-Lotem S, de Jong J, Meir $\mathrm{N}$ (eds) Methods for assessing multilingual children: disentangling bilingualism from language impairment, vol 35. Multilingual Matters, Bristol, pp 155-168

38. Hutson-Nechkash A (2001) Narrative toolbox: blueprints for story building. Eau Claire, Wl: Thinking Publications. Center for Speech and Language Pathology 12 (8): 648-895.

39. Ordóñez CL (2005) Oral bilingual proficiency of Colombian adolescents in an elite bilingual school. In: Cohen J, McAllister K, Rolstad K, MacSwan $J$ (eds) ISB4: Proceedings of the Fourth International Symposium on Bilingualism Somerville, vol 74. Cascadilla Press, pp 1765-1783

40. Rodina Y (2017) Narrative abilities of preschool bilingual NorwegianRussian children. Int J Biling 21(5):617-635

41. Merrikhi P (2012) The effect of "Bilingualism" on Iranian students' English grammar proficiency. Theory Pract Lang Stud 2(2):360-370

42. American Speech-Language-Hearing Association (ASHA) (2004) Bilingualism. knowledge and skills needed by speech-language pathologists and audiologists to provide culturally and linguistically appropriate services.

43. Altman C, Goldstein T, Armon-Lotem S (2017) Quantitative and qualitative differences in the lexical knowledge of monolingual and bilingual children. Clin Linguistic Phonol 31(11-12):931-954

44. Squires KE, Bedore LM, Lugo-Neris MJ, Pena ED, Bohman TM, Gillam RB (2014) Story retelling by bilingual children with language impairments and typically developing controls. Int J Lang Commun Disord 49(1):60-74

45. Klem M, Gustafsson JE, Hagtvet BE (2014) The dimensionality of language abilities in 4-year-olds, construct validation of a language screening tool. Scand J Educ Res 59(2):195-213

46. Armon-Lotem S, Gagarina N, Walters J (2011) The impact of internal and external factors on linguistic performance in the home language and in L2 among Russian-Hebrew and Russian-German preschool children. Linguistic Approaches to Bilingualism 1:291-317

47. Munoz DP, Everling S (2014) The anti-saccade task and the voluntary control of eye movement. Nat Rev Neurosci 5:218-228

48. Birdsong D (2005) Interpreting age effects in second language acquisition. In: Kroll J, DeGroot AMB (eds) Handbook of bilingualism; Psycholinguistic Prespective, vol 67. Oxford University Press, pp 109-127

49. Sheng L, Bedore LM, Peña ED, Fiestas C (2014) Semantic development in Spanish-English bilingual children. Effects of age and language experience. Child Dev 84(3):1034-1045

\section{Publisher's Note}

Springer Nature remains neutral with regard to jurisdictional claims in published maps and institutional affiliations.

\section{Submit your manuscript to a SpringerOpen ${ }^{\circ}$ journal and benefit from:}

- Convenient online submission

- Rigorous peer review

- Open access: articles freely available online

- High visibility within the field

- Retaining the copyright to your article

Submit your next manuscript at $\boldsymbol{\Delta}$ springeropen.com 